\title{
Production of Fe clusters by collisions of metal vapour with supersonic argon beams
}

\author{
Atea Akraiam and Klaus von Haeften \\ University of Leicester, Department of Physics and Astronomy, University Road, Leicester, LE1 7RH, United Kingdom \\ Received: date / Revised version: date

\begin{abstract}
The growth of Fe clusters by collisions of Fe atoms with Ar atoms flowing in a supersonic beam was investigated by Fe mass flux measurements and transmission electron (TEM) microscopy. Moderate Ar densities of the order of $1 \times 10^{20} \mathrm{~m}^{-3}$ were sufficient to cause cluster growth which was attributed to the low temperature of the Ar beam. TEM imaging of deposited clusters revealed diameter distributions from 2 to $10 \mathrm{~nm}$ depending on the deposition time. Extrapolation to zero deposition time revealed a cluster size of $2.4 \mathrm{~nm}$ grown in the gas phase. Growth on the surface was attributed to diffusion of single Fe atoms which are co-deposited with the clusters in the process and which agglomerate when they hit a cluster.
\end{abstract}

PACS. XX.XX.XX No PACS code given

\section{Introduction}

Metal clusters show unique properties; they are relevant to fundamental research of the structure of matter, in particular of how properties change with size, as well as various practical applications [1-5]. Molecular beams play an important role in the generation of metal clusters in the gas phase and in cluster beam deposition. Traditional molecular beam methods are supersonic expansion and gas aggregation [6-8]. They produce high intensities and provide convenient control of cluster size [9]. Supersonic beam sources for metal clusters require very high temperatures to establish sufficient vapour pressure and it is generally difficult to reach conditions for condensation in the expansion [10]. Therefore, they are predominantly suitable for the production of smaller clusters [9-12]. Condensation can be reinforced if an inert seed gas is added into the source volume and the mixture of seed gas and metal vapour is coexpanded $[13,14]$, however this approach is restricted to small concentrations of metal. The seed gas collisions need to be in excess of the metal-metal collisions, otherwise the effect of cooling during expansion into vacuum will be compromised and cause beam heating. Both the pure supersonic expansion and the seeded coexpansion sources produce metal clusters that are hot; in fact, they remain liquid during their entire journey through the molecular beam apparatus [15]. To date, Fe clusters have mostly been produced using gas aggregation sources in which the $\mathrm{Fe}$ is evaporated using either lasers [16], thermal heating [17] or arc erosion [18]. A notable exception is the supersonic source reported in [19] which works using the seed gas-co-expansion principle. However,

Send offprint requests to: the purpose of the source is to provide monoenergetic atomic Fe beams for lithography applications and the authors have not reported condensation in the gas phase yet.

To be able to vary cluster properties in the widest possible range control of the chemical composition and, ideally, of the geometrical structure is desirable. In addition, high flux rates are important since these are required for applications such as cluster-based thin film deposition [10-12,20-24]. We began exploring a new route of generating metal clusters by blowing supersonic rare gas beams into dilute metal vapours to provide the densities necessary for cluster growth. Despite using a supersonic beam, this approach has more similarities with the classical gas aggregation method [6], the main difference being the considerably lower temperatures of the aggregation medium. Typical gas aggregation applications employ argon and argon/helium mixtures cooled to $77 \mathrm{~K}$ by collisions with the chamber walls, whereas supersonic beams can reach much lower temperatures, sometimes even below $1 \mathrm{~K}$ [25]. It may therefore be possible to grow clusters in geometries far from thermal equilibrium, as has been demonstrated for $\mathrm{HCN}$ chains grown inside superfluid helium droplets into a head to tail configuration [26] or metal clusters with molecular type-electronic structure [27]. Furthermore, the use of rare gas supersonic beams has the potential to direct the metal clusters towards a substrate and form an intense beam.

In this paper we show the feasibility of this approach for iron clusters. Iron and iron oxide nanoparticles attract great interest because of their relevance as building blocks in magnetic materials [28], nanostructured data storage devices [19], as contrast agents for magnetic nuclear resonance imaging in medical diagnostics [29-31] or as magnetic absorbers in hyperthermia cancer therapy [32]. De- 
position rates of up to $5 \AA / \mathrm{min}$ were achieved at moderate stagnation pressures.

An important feature of our new approach is that the kinetic energy per atom of $\sim 50 \mathrm{meV}$ determined by the supersonic beam speed is significantly lower than typical bond energies in metals. Soft-deposition of metal clusters meaning the preservation of size and structure upon impact and interaction with the surface should be possible.

\section{Experiment}

The experiments were carried out in a molecular beam apparatus pumped by a baffled 12,000 l/s diffusion pump (DIP 12000, Leybold) and a $1600 \mathrm{l} / \mathrm{s}$ turbo molecular pump (Turbovac TW1600, Leybold). The baffle reduced the nominal pumping speed of the diffusion pump by approximately $50 \%$ [33]. The pumps were connected to the vessel on either end in order to be able to use a skimmer and pump two compartments separately. Here, no skimmer was used in order to maximise the deposition rates. The chamber was baked before each experimental run, after which a typical base pressure of $3 \cdot 10^{-9}$ mbar was measured.

Intense supersonic argon beams were generated using a conical nozzle of $100 \mu \mathrm{m}$ throat diameter and $20^{\circ}$ half opening angle ${ }^{1}$. This nozzle generates beam intensities that are eight times higher compared to an orifice of similar diameter [25]. The nozzle was mounted on a x-y-z translation stage. The stagnation pressure $\mathrm{P}_{0}$ was varied between 0.6 and 5 bar and the stagnation temperature $\mathrm{T}_{0}$ was $300 \mathrm{~K}$. Up to 5 bar the chamber pressure $\mathrm{P}_{1}$ increased linearly with a constant ratio of $\mathrm{P}_{0} / \mathrm{P}_{1}=\sim 1.4 \times 10^{6}$, showing that the pumping speed did not decrease. Under these conditions the beam is practically free of clusters as can be verified using well-known scaling laws for the condensation parameter $\Gamma^{*}$ with:

$$
\Gamma^{*}=k P_{0} d^{0.85} / T_{0}^{2.29}
$$

where $k=1646$ is a parameter for argon, $P_{0}$ the stagnation pressure in mbar, $d_{e q}$ the nozzle diameter in $\mu \mathrm{m}$ and $T_{0}$ the stagnation temperature. In this equation which has originally been developed for point-like orifices the diameter $d$ had been replaced by an equivalent diameter $d_{e q}$ that accounts for the specific properties of conical nozzles. The equivalent nozzle diameter $d_{e q}$ is larger than the throat diameter $d$ of the cone and the following relation holds:

$$
d_{e q}=0.74 \times d / \tan \alpha
$$

where $\tan \alpha$ is the half opening angle of the conical nozzle. The relation between average cluster size, N, and condensation parameter, $\Gamma^{*}$, is:

$$
N=33 \cdot\left(\Gamma^{*} / 1000\right)^{2.35}
$$

$[34,35]$. For 1 bar the average cluster sizes calculated with equations 1 and 3 is $2-3$ atoms.

1 This conical nozzle is of the same make as those employed in pulsed Even-Lavie sources.

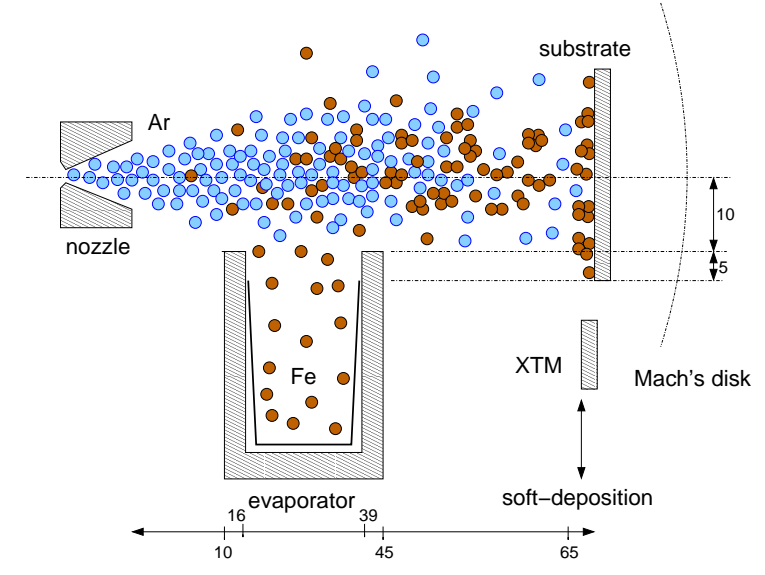

Fig. 1. Schematic showing the production of metallic nanoclusters by condensation of atomic metal vapours in supersonic rare gas beams. A conical nozzle on the left generates a supersonic argon beam which is blown into iron vapour. The collisions with Ar atoms cool the Fe atoms causing cluster formation and transfer forward momentum to the Fe atoms and clusters. The Fe clusters flow further downstream and are softdeposited with an impact energy of the order of $50 \mathrm{meV}$ per atom onto a substrate. The distance between nozzle and metal plume is shorter than the theoretical position of the Mach disk, therefore the entire process from cluster formation to deposition lies within a region of supersonic flow if the beam is clear of XTM or substrate holder. If the latter are moved into the beam, turbulences may occur (see text). All distances are in $\mathrm{mm}$. The Fe crucible has a conical shape; the diameter is about $10 \mathrm{~mm}$ at the bottom and $23 \mathrm{~mm}$ at the exit.

In our default configuration the supersonic beam passed above a thermal evaporator for $\mathrm{Fe}$ at a distance of $10 \mathrm{~mm}$, measured from the outer edge of the conical nozzle, where the argon atoms would collide with Fe atoms in a perpendicular manner. The vertical position of the evaporator was adjustable and set to a distance of $10 \mathrm{~mm}$ between the supersonic beam axis and the upper edge of a watercooled jacket, which was the highest part. The supersonic beam left the region of the Fe atom plume at a distance of $45 \mathrm{~mm}$. At $65 \mathrm{~mm}$ distance from the nozzle the Fe mass flow intensity was measured using a crystal thickness monitor (XTM)/micro balance, which was mounted on a linear drive so it could be moved in and out of the beam. Alternatively, graphite films (Agar S160) supported on copper grids for transmission electron microscope (TEM) analysis were moved into the beam to soft-deposit the Fe clusters. We anticipated that the entire process from cluster growth to deposition lay well within the region of supersonic flow because the theoretical distance between nozzle and Mach disk $x_{d}=0.67 d_{e q}\left(P_{0} / P_{1}\right)^{0.5}$ was 148 to $167 \mathrm{~mm}$, depending on the conditions. However, this estimate of the location of the Mach disk applies with some reservation. First, the equivalent nozzle diameter calculated with equation 2 is twice as large as the throat diameter. This contradicts the eight-times increased flux compared to an orifice reported by Even and coworkers [25]. Presumably, the equivalent nozzle diameter is significantly larger than the value given by equation 2 and the position of the Mach 
Table 1. Calculated Ar particle densities as a function of nozzle distance $x$. The Ar particle densities $n(x)$ were deduced using the equation for orifices $n(x)=0.157 n_{0}(d / x)^{2}$, where $n_{0}$ is the particle density before the nozzle, $d$ the nozzle diameter and $\mathrm{x}$ the distance from the nozzle. A correction factor of 8 taken from [25] was employed to account for the lower divergence of the conical nozzle compared to orifices. The chamber pressure $\mathrm{P}_{1}$ is given as well.

\begin{tabular}{lllll}
\hline $\begin{array}{l}\mathrm{P}_{0} \\
{[\text { bar }]}\end{array}$ & $\begin{array}{l}\mathrm{P}_{1} \\
{[\text { mbar }]}\end{array}$ & $\begin{array}{l}\mathrm{n}_{0} \\
\mathrm{~m}^{-3}\end{array}$ & $\begin{array}{l}\mathrm{n}_{x=20} \\
\mathrm{~m}^{-3}\end{array}$ & $\begin{array}{l}\mathrm{n}_{x=40} \\
\mathrm{~m}^{-3}\end{array}$ \\
\hline 0.6 & $5 \times 10^{-4}$ & $1.45 \times 10^{25}$ & $2.35 \times 10^{20}$ & $5.88 \times 10^{19}$ \\
1 & $8 \times 10^{-4}$ & $2.42 \times 10^{25}$ & $3.91 \times 10^{20}$ & $9.80 \times 10^{19}$ \\
1.6 & $1.3 \times 10^{-3}$ & $3.86 \times 10^{25}$ & $6.27 \times 10^{20}$ & $1.57 \times 10^{20}$ \\
2 & $1.5 \times 10^{-3}$ & $4.83 \times 10^{25}$ & $7.84 \times 10^{20}$ & $1.96 \times 10^{20}$ \\
2.6 & $1.8 \times 10^{-3}$ & $6.28 \times 10^{25}$ & $1.02 \times 10^{21}$ & $2.55 \times 10^{20}$ \\
3 & $2.1 \times 10^{-3}$ & $7.24 \times 10^{25}$ & $1.18 \times 10^{21}$ & $2.94 \times 10^{20}$ \\
3.6 & $2.4 \times 10^{-3}$ & $8.69 \times 10^{25}$ & $1.41 \times 10^{21}$ & $3.53 \times 10^{20}$ \\
4 & $2.7 \times 10^{-3}$ & $9.66 \times 10^{25}$ & $1.57 \times 10^{21}$ & $3.92 \times 10^{20}$ \\
4.6 & $3.0 \times 10^{-3}$ & $1.11 \times 10^{26}$ & $1.80 \times 10^{21}$ & $4.51 \times 10^{20}$ \\
5 & $3.5 \times 10^{-3}$ & $1.21 \times 10^{26}$ & $1.96 \times 10^{21}$ & $4.90 \times 10^{20}$ \\
\hline
\end{tabular}

disk should therefore be even farther from the nozzle. Second, equation 2 is only valid for unperturbed beams, and as we will see later this criterion is not fulfilled when the intensity is high and the XTM detector or the substrate holder are moved into the supersonic beam.

To better understand the formation process of metal clusters, the distances between nozzle and evaporator and between evaporator and XTM were varied. A schematic of the set-up including the relevant distances in the default configuration is shown in figure 1 .

To illustrate the cluster formation process, particle densities of Ar and Fe are provided in Table 1 and Table 2, respectively. The Fe particle densities along the intersection region with the supersonic beam are very difficult to determine. We therefore only show the densities corresponding to saturated vapour pressure. These densities are those that would be generated inside the crucible. In the vertical direction they drop; therefore, the values are only illustrative and serve as the ultimate upper limit for the density in the region where the metal vapour is intersected by the supersonic beam. Comparing the densities of iron and argon shows that the densities of argon are in excess of iron, which is a condition for efficient cooling.
Table 2. Fe particle densities as a function of temperature corresponding to the well-known vapour pressure curve.

\begin{tabular}{lll}
\hline $\mathrm{T}$ & $\mathrm{P}$ & $\mathrm{n}_{F e}$ \\
{$\left[{ }^{0} C\right]$} & {$[$ mbar $]$} & $\mathrm{m}^{-3}$ \\
\hline 727 & $1.33 \times 10^{-11}$ & $9.66 \times 10^{10}$ \\
777 & $1.33 \times 10^{-10}$ & $9.20 \times 10^{11}$ \\
832 & $1.33 \times 10^{-9}$ & $8.74 \times 10^{12}$ \\
892 & $1.33 \times 10^{-8}$ & $8.29 \times 10^{13}$ \\
957 & $1.33 \times 10^{-7}$ & $7.85 \times 10^{14}$ \\
1032 & $1.33 \times 10^{-6}$ & $7.40 \times 10^{15}$ \\
1127 & $1.33 \times 10^{-5}$ & $6.90 \times 10^{16}$ \\
1227 & $1.33 \times 10^{-4}$ & $6.44 \times 10^{17}$ \\
1342 & $1.33 \times 10^{-3}$ & $5.98 \times 10^{18}$ \\
1477 & $1.33 \times 10^{-2}$ & $5.52 \times 10^{19}$ \\
1647 & $1.33 \times 10^{-1}$ & $5.03 \times 10^{20}$ \\
1857 & $1.33 \times 10^{0}$ & $4.54 \times 10^{21}$ \\
2117 & $1.33 \times 10^{1}$ & $4.04 \times 10^{22}$ \\
2467 & $1.33 \times 10^{2}$ & $3.53 \times 10^{23}$ \\
2927 & $1.33 \times 10^{3}$ & $3.02 \times 10^{24}$ \\
\hline
\end{tabular}

Fe was evaporated using a home-made thermal evaporator (design similar to WEZ 40-10KS, Eberl GmbH) that consisted of a central crucible, a cylindrical arrangement of tantalum filaments surrounded by tantalum heat shields and a stainless steel water-cooled shroud. Fe pellets of purity grade $99.95 \%$ (Lesker) were loaded in an $\mathrm{Al}_{2} \mathrm{O}_{3}$ crucible. To reduce contamination with impurities, the empty crucible was baked in vacuum before it was loaded with Fe. After loading the crucible the vacuum chamber was baked again. The evaporation temperature was measured using a K-type thermocouple, but we found that these measurements were offset by up to $200^{\circ} \mathrm{K}$ because we observed that the Fe had melted inside the crucible when the thermocouple measured a temperature $200{ }^{0} \mathrm{C}$ lower than the melting temperature of $1410{ }^{\circ} \mathrm{C}$. Therefore, we state in the following the heating voltage rather than the crucible temperature.

Fe beam intensities were measured using the XTM as a function of nozzle-evaporator distance, of XTM-evaporator distance, argon stagnation pressure $P_{0}$ and heating voltage of the Fe evaporator. These intensities relate to the total mass flux and contain condensed and uncondensed Fe. Furthermore, Fe clusters were deposited on the graphitecoated copper grids for different Fe deposition times while the stagnation pressure and evaporator voltage where held 


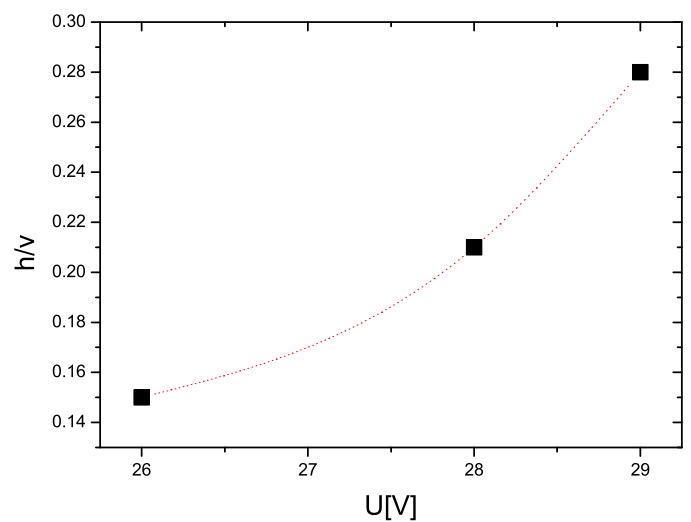

Fig. 2. Ratio of the Fe deposition rates measured with the XTM mounted horizontally with respect to the supersonic beam and vertically above the crucible as a function of the evaporation voltage. The nozzle-evaporator distance was $18 \mathrm{~mm}$ and the evaporator-horizontal-XTM distance was $22 \mathrm{~mm}$. The distance between the vertical XTM and the upper edge of the water shroud of the evaporator was $33 \mathrm{~mm}$. All parameters of these measurements are listed in table 3 . The dotted line is a guide to the eye.

unchanged: images were recorded using TEM. The TEM images were analysed in a semi-automatic procedure using IMAGEJ. This software draws a contour line around the clusters connecting points of equal intensity, and calculates the enclosed area $A$ for each cluster. In the last step a distribution function of the cluster diameter $d$ is produced, where $d$ is deduced from circles of areas $A=\pi d^{2} / 4$ similar to those of the clusters identified by IMAGEJ.

\section{Results and Discussion}

\subsection{XTM measurements of Fe flux}

Typical Fe deposition rates are shown in table 3. Deposition rates were measured in the direction of the supersonic beam axis (horizontal) and along the axis of the Fe crucible (vertical), i.e. the XTM was placed above the crucible to measure the Knudsen flow of Fe atoms. The rates in the vertical direction were recorded with the Ar beam off, the rates in the horizontal direction with Ar beam on. Thus, the ratio between these two values provides a measure of how efficiently the upwards directed Fe flow is deflected horizontally. Table 3 and figure 2 show these rates as a function of the evaporation voltage. Although the stagnation pressure is kept constant an increase of the horizontal/vertical rate is observed when the heating voltage is increased. We show here that in the limit of Ar density in excess of $\mathrm{Fe}$ - which is always fulfilled in our experiment - such an increase is evidence of cluster formation. If the only effect of increasing the crucible temperature were an increase of the Fe atom density without condensation the ratio between horizontal and vertical

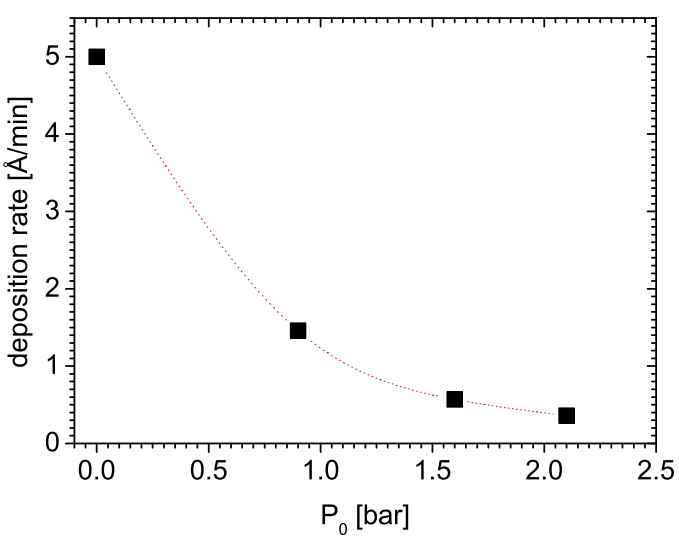

Fig. 3. Deposition rate measured with the XTM mounted in the vertical direction as a function of the stagnation pressure. The flux in vertical direction drops considerably when the supersonic flux is increased. The dotted line is a guide to the eye.

Table 3. Typical parameters. The Fe deposition rate in vertical direction was measured with Ar beam off. The deposition rate in horizontal direction was measured with Ar beam on

\begin{tabular}{|c|c|c|c|c|c|c|}
\hline $\begin{array}{l}\mathrm{U} \\
{[V]}\end{array}$ & $\begin{array}{l}\mathrm{P} \\
{[W]}\end{array}$ & $\begin{array}{l}\mathrm{T} \\
{\left[{ }^{0} \mathrm{C}\right]}\end{array}$ & $\begin{array}{l}\mathrm{P}_{0} \\
{[\text { bar }]}\end{array}$ & $\begin{array}{l}\text { hor. } \\
{[\AA / \min ]}\end{array}$ & $\begin{array}{l}\text { vert. } \\
{[\AA / \mathrm{min}]}\end{array}$ & ratio $\mathrm{h} / \mathrm{v}$ \\
\hline 26 & 135 & 1137 & 1.0 & 0.71 & 4.6 & 0.15 \\
\hline 28 & 153 & 1187 & 1.0 & 2.5 & 12 & 0.21 \\
\hline 29 & 163 & 1213 & 1.0 & 5.5 & 20 & 0.28 \\
\hline
\end{tabular}

deposition would be unchanged. An increase in the collisional cross section due to cluster growth increases the number of collisions per particle which has two effects: (i) the Fe particles are slowed down and dwell longer in the aggregation region; (ii) as a consequence the particles accumulate increasingly more momentum in horizontal direction. The observed increase of the horizontal/vertical deposition rate ratio therefore provides evidence that the increase of the heating voltage from 26 to $29 \mathrm{~V}$ produced larger clusters and that this increased growth is caused by higher Fe densities in the aggregation region.

Figure 3 shows the Fe flux measured in the vertical direction as a function of the supersonic beam intensity. Blowing Ar gas sidewards into Fe vapour redirects momentum from the vertical to the horizontal direction and depletes the signal picked up by the vertical XTM. Already at 1 bar stagnation pressure the Fe flow in vertical direction has decreased to about 20\%. Further increase of the stagnation pressure beyond 2 bar drops the vertical flow to less than $10 \%$ of the value without the supersonic beam on. This behaviour can be entirely explained by the increase of the number of collisions leading to forward momentum transfer. An important consequence is that compared to other deposition methods extremely small amounts of material are wasted. This feature will be important for applications that use precious metals. 
Table 4. Fe deposition rate measured in the vertical direction as a function of the stagnation pressure for $26 \mathrm{~V}$ heating voltage.

\begin{tabular}{ll}
\hline $\mathrm{P}_{0}$ & vert. \\
{$[$ bar $]$} & {$[\AA ̊ \mathrm{~min}]$} \\
\hline 0 & 5.0 \\
0.9 & 1.46 \\
1.6 & 0.57 \\
2.1 & 0.36
\end{tabular}

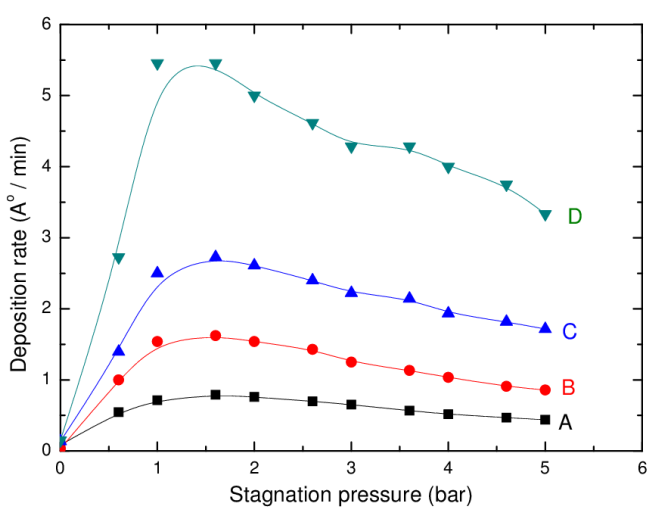

Fig. 4. Fe deposition rates as a function of Ar stagnation pressure for different Fe heating voltages. The data sets labelled with $\mathrm{A}, \mathrm{B}, \mathrm{C}$, and $\mathrm{D}$ belong to $26,27,28$ and $29 \mathrm{~V}$ heating voltages, respectively. The nozzle throat-evaporator distance was $18 \mathrm{~mm}$, the evaporator-XTM distance was $22 \mathrm{~mm}$ and the evaporator diameter was $30 \mathrm{~mm}$.

Variation of the Fe deposition rate with supersonic beam intensity, i.e. the Ar-Fe collision rate, was investigated by XTM measurements in the horizontal direction. Figure 4 shows $\mathrm{Fe}$ deposition rates as a function of the stagnation pressure, $\mathrm{P}_{0}$, measured using the XTM for very short beam lengths $(70 \mathrm{~mm})$. In this configuration, deposition rates up to $5.5 \AA / \mathrm{min}$ were measured. The stagnation pressure dependence of the deposition rate was measured for several heating voltages $(26,27,28$ and $29 \mathrm{~V}$ ), all showing the same trends: for small stagnation pressures less than 1 bar the deposition rate increased linearly with stagnation pressure; above 1 bar, the deposition rate increased less rapidly, passed through a maximum at 1.6 bar and decreased thereafter.

The increase in Fe deposition rate with increasing $\mathrm{Ar}$ flux in the region below $\mathrm{P}_{0}=1.6$ bar clearly shows the effect of forward momentum transfer to the Fe atoms. The drop in Fe forward beam intensity at stagnation pressures above $\mathrm{P}_{0}=1.6$ bar is rather unexpected, but the fact that it is correlated with the Ar flux shows that at $\mathrm{P}_{0}=1.6$ bar, the supersonic beam seems to reach a critical intensity. It is quite possible that turbulences and shock waves are produced when the XTM surface is hit at normal incidence.

To verify the hypothesised decrease in deposition rate caused by perturbation of the supersonic beam we in-
Table 5. Supersonic cluster beam deposition rates reported in the literature.

\begin{tabular}{llll}
\hline $\begin{array}{l}\text { deposition } \\
\text { rate }[\mathrm{nm} / \mathrm{s}]\end{array}$ & $\begin{array}{l}\text { distance } \\
\text { from nozzle }\end{array}$ & comment & reference \\
\hline 0.04 & n.s. & $\mathrm{Ag}_{n}$ & {$[8]$} \\
0.01 & n.s. & $\mathrm{Ag}_{n}$ & {$[7]$} \\
2000 & $60 \mathrm{~mm}$ & $\mathrm{Cs}_{n}, \mathrm{~N}=2500$ & {$[12]$} \\
100 & $300 \mathrm{~mm}$ & $\mathrm{Zn}_{n}$ & {$[23]$} \\
1.8 & $479 \mathrm{~mm}$ & $\mathrm{Ag}_{n}, \mathrm{Ar}$ coexp. & {$[11]$} \\
10 & $120 \mathrm{~mm}$ & $\mathrm{Ag}_{n}, \mathrm{Ar}$ coexp. & {$[24]$} \\
0.01 & $700 \mathrm{~mm}$ & $\mathrm{Fe}_{n}, \mathrm{Ar}$ beam & this work \\
\hline
\end{tabular}

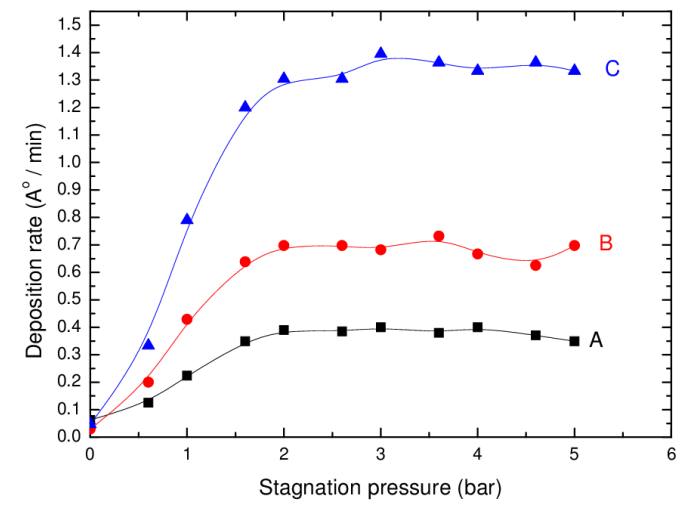

Fig. 5. Fe deposition rates measured in the horizontal direction as a function of Ar stagnation pressure for a long supersonic beam length to reduce interferences caused by the XTM. The data sets A, B, and C belong to heating voltages of 27,28 and $29 \mathrm{~V}$ respectively. The distance between nozzle and evaporator was $18 \mathrm{~mm}$ and the distance between evaporator and XTM was $82 \mathrm{~mm}$.

creased the XTM-evaporator distance to $82 \mathrm{~mm}$, so that the total supersonic beam length was increased from $40 \mathrm{~mm}$ to $100 \mathrm{~mm}$. Figure 5 shows Fe deposition rates as a function of stagnation pressure for different heating voltages $(27,28$ and $29 \mathrm{~V}$ ) in this configuration (evaporator-XTM distance of $82 \mathrm{~mm}$ ). Compared to figure 4 the rates are decreased because of the longer evaporator-XTM distance. Now, the maximum Fe deposition rates were measured for higher stagnation pressures at $\mathrm{P}_{0}=2-3$ bar, confirming our hypothesis. 
Specially shaped substrates or planar substrates introduced under an angle might reduce the effect of turbulences and make higher deposition rates possible. Table 5 compares the deposition rates achieved in this work with data reported in the literature. The highest possible deposition rates are obtained using pure and seeded supersonic beam sources. Our deposition rates are comparable to those reported in gas aggregation experiments $[7,8]$.

\subsection{TEM of deposited clusters}

Figure 6 shows TEM images of clusters deposited over 5 minutes, 10 minutes and 15 minutes at $\mathrm{P}_{0}=1$ bar, $\mathrm{T}_{0}=329 \mathrm{~K}$ and $\mathrm{V}=28 \mathrm{~V}$. Equivalent diameters $d$ were deduced from the TEM for each cluster using IMAGEJ; their distribution functions are shown in figure 7 for different deposition times. Average cluster diameters are shown in figure 7 as a function of deposition time.

Despite all parameters that are expected to determine growth in the gas phase (Fe density and Ar beam intensity) being kept constant, the TEM images show that the cluster sizes increase with deposition time. Hence, the increase in cluster size must be related to growth on the surface after the clusters have been deposited. The simplest explanation is that uncondensed Fe atoms that are carried with the supersonic beam onto the surface diffuse across it until they eventually hit a cluster and agglomerate. The amount of single Fe atoms on the surface increases linearly with decreasing deposition time. Assuming lateral growth, one would expect the cluster size to increase with the square root of the deposition time. This interpretation is supported by the widths of the distribution functions which increase with deposition time. Increased average sizes after longer deposition have been reported for neutral Ag clusters deposited on amorphous carbon $[7,8]$.

Figure 8 shows the average diameter determined from the distribution in figure 7 as a function of deposition time as well as a fit of a square-root function accounting for two-dimensional growth. The intercept of the fit with the ordinate yields the cluster diameter at zero deposition time, i.e. the size of the clusters in the gas phase, for which we obtain a diameter of $2.4 \mathrm{~nm}$.

The clusters in all three TEM images in figure 6 appear randomly distributed rather then grouped $[36,37]$ or ordered [38]. Possibly, $2.4 \mathrm{~nm}$ sized Fe clusters do not move much, or not at all after deposition. Also, the clusters have circular or square-like shapes rather than ramified islands, as has been seen for $\mathrm{Au}, \mathrm{Pt}, \mathrm{Co}$ and alloys of these clusters $[39,40]$. All clusters in this study have, after preparation, been transfered to the TEM in ambient air and are therefore expected to be oxidised. The TEM images in the middle and lower panel in figure 6 show higher contrast in the centre of the clusters, which we attribute to an $\mathrm{Fe}$ core. The image in the upper panel shows homogeneous contrast, which we attribute to fully oxidised clusters.
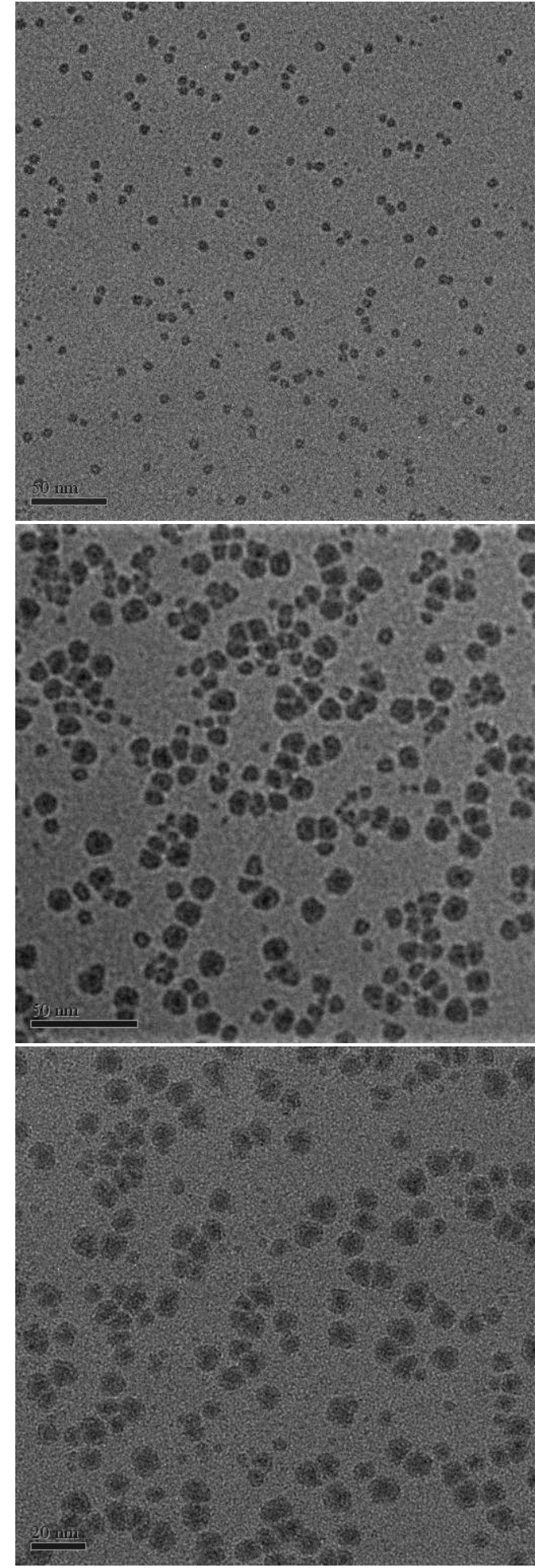

Fig. 6. TEM images of Fe clusters produced by blowing a supersonic Ar beam into Fe vapour. The deposition time was 5 minute for the upper, 10 minute for the middle and 15 minutes for the lower panel. All other parameters were unchanged. 


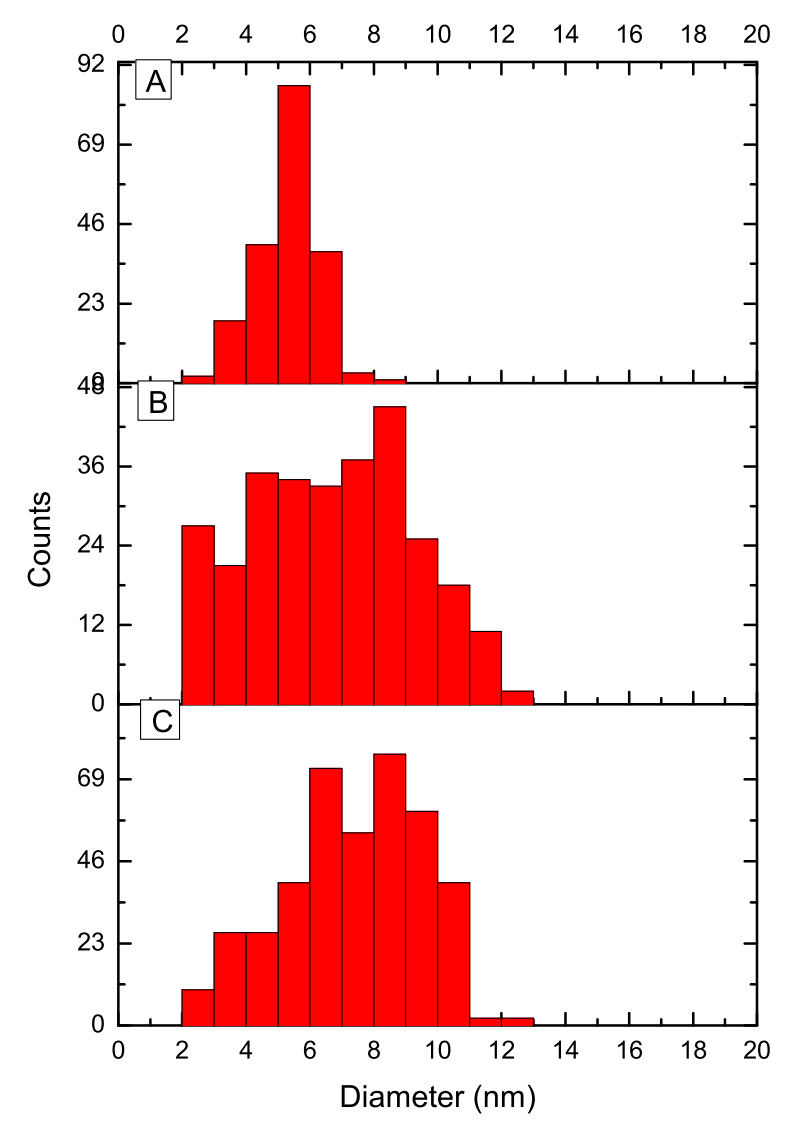

Fig. 7. Distribution of the diameter of Fe clusters for deposition times of (A) 5 minutes, (B) 10 minutes and (C) 15 minutes.

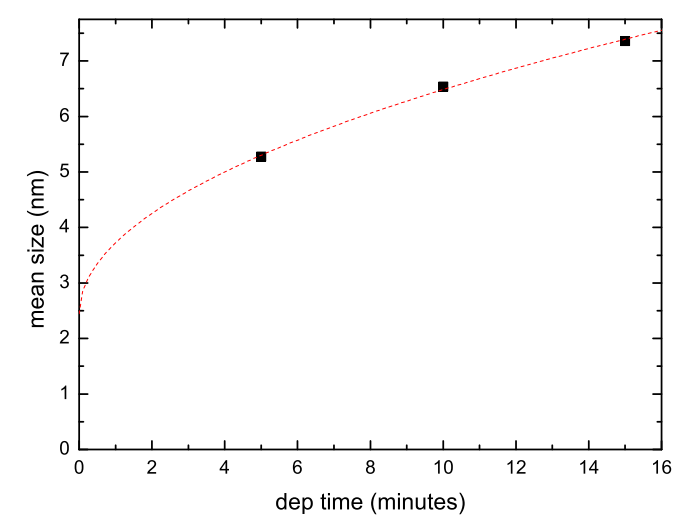

Fig. 8. Mean Fe cluster diameters as a function of deposition time. The increase with deposition time shows that cluster growth due to diffusion and agglomeration on the surface takes place. The intercept of the fit provides a measure of the cluster size in vacuum.

\section{Conclusions}

In conclusion, an intense supersonic beam was blown into Fe vapour and Fe cluster growth was observed. The Ar densities of the supersonic beam at the interaction point were one to two orders of magnitude lower those used in common gas aggregation cluster sources, showing that the process of cluster growth was very efficient, possibly because of the lower temperature of supersonic beams compared to traditional sources. Also, very small Ar clusters that were present in the beams under investigation could have enhanced growth.

XTM measurements of the vertical and horizontal Fe flux showed that the Fe cluster size depended on the Fe density. The XTM measurements have also confirmed that the deflection of vertically evaporated Fe into horizontal flow is very efficient. The presence of a moderate supersonic Ar beam decreased the vertical flux to less than 10\%, which is very encouraging for future applications where precious metals need to be deposited and waste has to be kept at a minimum.

The size of clusters was determined using TEM and found to depend on the deposition time showing that the clusters also grew on the substrate. We presume that single Fe atoms were deflected and carried with the supersonic beam alongside the clusters towards the substrate. After co-deposition, the Fe diffused on the surface and agglomerated with the clusters that were grown in the gas phase. Combining growth in the gas phase and on surfaces might be relevant for the selective production of specific nanostructures. For example, hat-shaped clusters with a 2D perimeter might be grown. Conversely, contribution of atoms to growth can be suppressed by the use of aerodynamic lenses because their throughput is size-selective $[5]$.

Finally, we anticipate that our study is relevant for the interpretation of droplet/cluster experiments where clusters are grown by the pick-up method [41,42], in particular where the metal vapour densities are high and the metal cluster sizes are large [43,44]. Helium droplet beams are always accompanied by a fraction of small clusters and uncondensed atoms [45]. It is possible that condensation of metal vapours is triggered by inelastic collisions with such small helium clusters or in elastic collisions with the atoms of the uncondensed part of the beam. Our study helps understanding of these effects.

\section{References}

1. W.A. de Heer, Rev. Mod. Phys. 65(3), 611 (1993). DOI 10.1103/RevModPhys.65.611

2. U. Kreibig, M. Vollmer, Optical properties metal clusters (Springer-Verlag, 1995)

3. C. Binns, Surf. Sci. Rep. 44(1-2), 1 (2001)

4. F. Baletto, R. Ferrando, Rev.s Modern Phys. $77(1), \quad 371 \quad(2005) . \quad$ URL http://link.aps.org/abstract/RMP/v77/p371

5. K. Wegner, P. Piseri, H. Tafreshi, P. Milani, J. Phys. D: Appl. Phys. 39(22), R439 (2006) 
6. K. Sattler, J. Mühlbach, E. Recknagel, Phys. Rev. Lett. 45(10), $821(1980)$

7. F. Frank, W. Schulze, B. Tesche, J. Urban, B. Winter, Surface Science 156, 90 (1985)

8. J. Urban, B. Tesche, Thin solid films 137(2), 325 (1986)

9. O. Hagena, Zeitschrift für Physik D Atoms, Molecules and Clusters 4(3), 291 (1987)

10. O. Hagena, Zeitschrift für Physik D Atoms, Molecules and Clusters 17(3), 157 (1990)

11. O. Hagena, Zeitschrift für Physik D Atoms, Molecules and Clusters 20(1), 425 (1991)

12. J. Gspann, Zeitschrift für Physik D Atoms, Molecules and Clusters 20(1), 421 (1991)

13. M. Kappes, R. Kunz, E. Schumacher, Chem. Phys. Lett. 91(6), 413 (1982)

14. W. Knight, K. Clemenger, W. de Heer, W. Saunders, M. Chou, M. Cohen, Phys. Rev. Lett. 52(24), 2141 (1984)

15. J. Gspann, Zeitschrift für Physik D Atoms, Molecules and Clusters 3(2), 143 (1986)

16. M. Sakurai, K. Watanabe, K. Sumiyama, K. Suzuki, J. chemical physics 111, 235 (1999)

17. K. Edmonds, C. Binns, S. Baker, S. Thornton, C. Norris, J. Goedkoop, M. Finazzi, N. Brookes, Phys. Rev. B 60(1), 472 (1999)

18. R.P. Methling, V. Senz, E.D. Klinkenberg, T. Diederich, J. Tiggesbäumker, G. Holzhüter, J. Bansmann, K.H. Meiwes-Broer, Eur. Phys. J. D 16(1), 173 (2001)

19. R. Bosch, H. Beijerinck, P. Van der Straten, K. van Leeuwen, European Phys. J. Appl. Phys. 18(03), 221 (2002)

20. I. Yamada, T. Takagi, Thin Solid Films 80(1), 105 (1981)

21. J. Gspann, Nuclear Instruments and Methods in Phys. Research Section B: Beam Interactions with Materials and Atoms 37, 775 (1989)

22. J. Gspann, Nuclear Instruments and Methods in Phys. Research Section B: Beam Interactions with Materials and Atoms 80, 1336 (1993)

23. J. Gspann, Zeitschrift für Physik D Atoms, Molecules and Clusters 26, 174 (1993)

24. P. Gatz, O. Hagena, Appl. surface science 91(1), 169 (1995)

25. U. Even, J. Jortner, D. Noy, N. Lavie, C. Cossart-Magos, J. Chem. Phys. 112, 8068 (2000)

26. K. Nauta, R.E. Miller, Science 283(5409), 1895 (1999)

27. A. Bartelt, J.D. Close, F. Federmann, N. Quaas, J.P. Toennies, Phys. Rev. Lett. 77(17), 3525 (1996). URL http://link.aps.org/abstract/PRL/v77/p3525

28. G. Chaubey, V. Nandwana, N. Poudyal, C. Rong, J. Liu, Chemistry Materials 20(2), 475 (2007)

29. L. Babes, B. Denizot, G. Tanguy, J. Le Jeune, P. Jallet, J. Colloid and Interface Science 212(2), 474 (1999)

30. A. Gupta, M. Gupta, Biomaterials 26(18), 3995 (2005)

31. C. Hadjipanayis, M. Bonder, S. Balakrishnan, X. Wang, H. Mao, G. Hadjipanayis, Small 4(11), 1925 (2008)

32. K. Hayashi, M. Moriya, W. Sakamoto, T. Yogo, Chemistry Materials 21(7), 1318 (2009)

33. K. von Haeften, S. Rudolph, I. Simanovski, M. Havenith, R. Zillich, K. Whaley, Phys. Rev. B 73(5), 054502 (2006). URL http://link.aps.org/abstract/PRB/v73/e054502

34. O.F. Hagena, W. Obert, J. Chem. Phys. 56(5), 1793 (1972). URL http://link.aip.org/link/?JCP/56/1793/1

35. R. Karnbach, M. Joppien, J. Stapelfeldt, J. Wörmer, T. Möller, Rev. Sci. Instrum. 64, 2838 (1993)
36. L. Bardotti, F. Tournus, P. Mélinon, M. Pellarin, M. Broyer, Phys. Rev. B 83(3), 035425 (2011)

37. F. Tournus, L. Bardotti, V. Dupuis, J. Appl. Phys. 109, 114309 (2011)

38. R. Alayan, L. Arnaud, M. Broyer, E. Cottancin, J. Lerme, S. Marhaba, J. Vialle, M. Pellarin, Phys. Rev. B 76(7), 075424 (2007)

39. R. Alayan, L. Arnaud, M. Broyer, E. Cottancin, J. Lermé, J. Vialle, M. Pellarin, Phys. Rev. B 73(12), 125444 (2006)

40. L. Bardotti, F. Tournus, M. Pellarin, M. Broyer, P. Mélinon, V. Dupuis, Surface Science 606(1), 110 (2012)

41. T.E. Gough, M. Mengel, P.A. Rowntree, G. Scoles, J. Chem. Phys. 83(10), 4958 (1985). URL http://link.aip.org/link/?JCP/83/4958/1

42. M. Lewerenz, B. Schilling, J.P. Toennies, J. Chem. Phys. 102(20), 8191 (1995). URL http://link.aip.org/link/?JCP/102/8191/1

43. T. Diederich, T. Döppner, J. Braune, J. Tiggesbäumker, K.H. Meiwes-Broer, Phys. Rev. Lett. 86, 4807 (2001). DOI 10.1103/PhysRevLett.86.4807

44. V. Mozhayskiy, M. Slipchenko, V. Adamchuk, A. Vilesov, J. Chem. Phys. 127, 094701 (2007)

45. K. von Haeften, T. Laarmann, H. Wabnitz, T. Möller, K. Fink, J. Phys. Chem. A 25(115), 7316 (2011). DOI 10.1021/jp2008489. URL http://pubs.acs.org/doi/abs/10.1021/jp2008489 